\title{
Systemic chemotherapy of pediatric recurrent ependymomas: results from the German HIT-REZ studies
}

\author{
Jonas E. Adolph ${ }^{1}$ (1) - Gudrun Fleischhack ${ }^{1}$ (1) Christine Gaab ${ }^{1} \cdot$ Ruth Mikasch ${ }^{1} \cdot$ Martin Mynarek $^{2}$. \\ Stefan Rutkowski ${ }^{2}$. Ulrich Schüller ${ }^{2,3,4}$ (D) Stefan M. Pfister ${ }^{5,6,7} \cdot$ Kristian W. Pajtler $^{5,6,7}$ - Till Milde ${ }^{5,6,7,8}$. \\ Olaf Witt $^{5,6,7,8} \cdot$ Brigitte Bison $^{9} \cdot$ Monika Warmuth-Metz $^{10} \cdot$ Rolf-Dieter Kortmann $^{11}$. Stefan Dietzsch ${ }^{11,13}$. \\ Torsten Pietsch $^{12}$ - Beate Timmermann ${ }^{13}$. Stephan Tippelt ${ }^{1}$ (D) - for the German GPOH HIT-Network
}

Received: 19 August 2021 / Accepted: 5 October 2021 / Published online: 16 October 2021

(c) The Author(s) 2021

\begin{abstract}
Purpose Survival in recurrent ependymoma (EPN) depends mainly on the extent of resection achieved. When complete resection is not feasible, chemotherapy is often used to extend progression-free and overall survival. However, no consistent effect of chemotherapy on survival has been found in patients with recurrent EPN.

Methods Systemic chemotherapeutic treatment of 138 patients enrolled in the German HIT-REZ-studies was analyzed. Survival depending on the use of chemotherapy, disease-stabilization rates (RR), duration of response (DOR) and time to progression (TTP) were estimated.

Results Median age at first recurrence was 7.6 years (IQR: 4.0-13.6). At first recurrence, median PFS and OS were 15.3 (CI 13.3-20.0) and 36.9 months (CI 29.7-53.4), respectively. The Hazard Ratio for the use of chemotherapy in local recurrences in a time-dependent Cox-regression analysis was 0.99 (CI 0.74-1.33). Evaluable responses for 140 applied chemotherapies were analyzed, of which sirolimus showed the best RR (50\%) and longest median TTP [11.51 (CI 3.98; 14.0) months] in nine patients, with the strongest impact found when sirolimus was used as a monotherapy. Seven patients with progression-free survival $>12$ months after subtotal/no-resection facilitated by chemotherapy were found. No definitive survival advantage for any drug in a specific molecularly defined EPN type was found.

Conclusion No survival advantage for the general use of chemotherapy in recurrent EPN was found. In cases with incomplete resection, chemotherapy was able to extend survival in individual cases. Sirolimus showed the best RR, DOR and TTP out of all drugs analyzed and may warrant further investigation.
\end{abstract}

Keywords Ependymoma $\cdot$ Chemotherapy $\cdot$ Recurrence $\cdot$ Children $\cdot$ Sirolimus

Stephan Tippelt

stephan.tippelt@uk-essen.de

1 Department of Pediatrics III, Center for Translational Neuroand Behavioral Sciences (CTNBS), University Hospital of Essen, Hufelandstraße 55, 45147 Essen, Germany

2 Department of Pediatric Hematology and Oncology, Center for Obstetrics and Pediatrics, University Medical Center Hamburg-Eppendorf, Hamburg, Germany

3 Institute of Neuropathology, University Medical Center Hamburg-Eppendorf, Hamburg, Germany

4 Research Institute Children's Cancer Center Hamburg, Hamburg, Germany

5 Hopp Children's Cancer Center Heidelberg (KiTZ), Heidelberg, Germany

6 Division of Pediatric Neurooncology, German Cancer Research Center (DKFZ), Heidelberg, Germany
7 Clinical Cooperation Unit Pediatric Oncology, German Cancer Research Center (DKFZ) and German Consortium for Translational Cancer Research (DKTK), Heidelberg, Germany

8 Department of Pediatric Oncology and Hematology, Heidelberg University Hospital, Heidelberg, Germany

9 Diagnostic and Interventional Neuroradiology, University Hospital Augsburg, Augsburg, Germany

10 Institute of Diagnostic and Interventional Neuroradiology, University of Würzburg, Würzburg, Germany

11 Department of Radio-Oncology, University of Leipzig, Leipzig, Germany

12 Institute of Neuropathology, DGNN Brain Tumor Reference Center, University of Bonn, Bonn, Germany

13 Clinic for Particle Therapy, West German Proton Therapy Center, University of Essen, Essen, Germany 


\section{Introduction}

Recurrent ependymomas (EPN) in children and adolescents feature a poor prognosis, with a median survival after diagnosis of recurrence of only 12 months [1,2]. Out of all patients diagnosed with a WHO grade II or III EPN, around $40 \%$ experience a progression or a relapse [3, 4].

While survival-benefits for surgery and radiotherapy have been shown in multiple cohorts of recurrent EPN, results on chemotherapy were less favorable $[1,5-8]$. So far, no uniform chemotherapeutic treatment regimen for recurrent EPN has been established. Results on the efficacy of individual drugs and specific combinations have been almost entirely restricted to trials with limited casenumbers $[9,10]$.

With advances in molecular characterization emerging in recent years, molecularly defined types of EPN are increasingly treated as distinct diseases, with the infratentorial PF-A and supratentorial ZFTA subtype being the most aggressive and most abundant in recurrences [11-13]. However, most trials on chemotherapy in recurrent EPN were done prior to these findings. Therefore, results on the efficacy in specific EPN types are still lacking. Recent molecular analyses have also suggested that EPN in general may possess features making them especially resistant to chemotherapy $[14,15]$, pointing towards the importance of finding chemotherapy-regimens that work specifically for EPN.

Here we report on a pooled cohort of patients with recurrent EPN from the multi-institutional HIT-REZstudies. We examine the influence of chemotherapy on survival and report on cases in which its use showed advantages. Furthermore, we examine the responses to different chemotherapy regimens and analyze them accounting for specific EPN types.

\section{Methods}

\section{Clinical trials}

The HIT-REZ-studies consisted of two multi-institutional trials [HIT-REZ 97 and HIT-REZ 2005 (NCT00749723)], as well as an ongoing registry (HIT-REZ registry) concerning recurrent CNS-tumors in children. Data was gathered through a centralized reporting system. Information included dates of recurrences, metastatic stage, extent of resection, as well as target volume and doses of radiotherapy.

Tumor response was measured through centralized assessment of MRI. An assessment of the response to chemotherapy could only be made if either at least one lesion was able to be clearly measurable in three dimensions, a non-measurable lesion was present or if malignant cells were detectable in the cerebrospinal fluid (CSF) before chemotherapy was applied. If a preceding surgery led to a complete resection of the tumor, only the time to next progression was measured. If a lesion could be defined, complete remission (CR) was defined as a complete absence of all lesions and malignant cells in CSF after systemic treatment. Partial remission (PR) was defined as a decrease in tumor volume of measurable lesion(s) by at least $50 \%$. Stable diseases (SD) were cases of a decrease in volume of less than $50 \%$ and no more than $25 \%$ of volume-gain or cases with persistent presence of non-measurable lesions. Progressive disease (PD) was chosen when MRI showed an increase in volume of at least $25 \%$ or occurrence of new measurable/non-measurable lesion(s) or new detection of tumor cells in the CSF.

Extent of resection was determined by postoperative MRI, with gross-total resection (GTR) being defined as no visible residual tumor. Near-total resection (NTR) was defined as enhancement at the edge of the resection area and a reduction in volume of at least $90 \%$. Surgery with less reduction in tumor volume was defined as a subtotal resection (STR), or as biopsy if no more than $10 \%$ was removed.

\section{Statistical analysis}

Response-rates (RR) were defined as the rate of CR, PR and SD being achieved through chemotherapy, while the objective response-rate (ORR) included only CR and PR. If surgery preceded chemotherapy in case of local or unifocal relapse, the residual tumor had to be measurable in three dimensions by MRI before chemotherapy was started to evaluate response. The duration of response (DOR) was defined as the time from the beginning of chemotherapy until the latest date of imaging in which the best grade of response could be found. If PD was the only response found for a chemotherapy regimen, DOR was set to zero months. The time to progression (TTP) was defined as the time from the start of chemotherapy to the time-point a progression was found on MRI and/or new tumor cells were detected in CSF cytology. Recurrences without measurable tumor residual after surgery were only evaluated as to TTP.

Overall survival (OS) was defined as the time from first recurrence to either death from any cause or to the last timepoint of follow-up. Progression-free survival (PFS) was defined as the time to next recurrence, death or last followup from prior recurrence. For cases of last follow-up censoring was used. Both OS and PFS were given as a median with its accompanying 95\% confidence interval (CI). For descriptive statistics of the study cohort, medians were given with their interquartile ranges (IQR) if not differently specified. 
Cox-regressions were used to examine the effect of specific covariates on either PFS or OS, as specified. Timedependent Cox-regressions were used for covariates subject to change during the studies and the follow-up, with the date of diagnosis of the first recurrence was taken as a starting point. Further recurrences as well as death were treated as cumulative events. Results of these Cox-regressions were calculated as a hazard ratio (HR) towards either OS or PFS and its 95\%-CI. P values for the HR were given in the calculation of the efficacy of different drugs depending on the molecular subgroup, with the a-value set at 0.05 .

All statistical analyses were done using $\mathrm{R}$ version 4.0.3 [16], using the survival package for all survival analyses and ggplot2 to produce all figures. The results section of this paper was compiled using R Markdown [17].

\section{Results}

138 patients with recurrent EPN (WHO grade II or III) from the HIT-REZ studies diagnosed between 1998 and 2018 were included in this analysis. They were followed up for a median of 31.6 months (IQR: 16.7-58.1, range: 2.3-197.4) and for a total number of 335 recurrences. Table 1 displays an overview of the characteristics of the analyzed cohort. Patients were predominantly male $(65.2 \%)$ and had a median age of 7.6 years (IQR: 4.0-13.6, range: 0.8-28.8) at first recurrence. EPN were mostly of infratentorial origin (74.6\%). Molecular classification was available for $64.5 \%$ of cases, of which $74.2 \%$ were PF-A and $20.2 \%$ ST-ZFTA.

\section{Survival}

Median OS from first recurrence was 36.9 months (CI 29.7-53.4). Median PFS from first recurrence was 15.3 months (CI 13.3-20.0). To analyze whether chemotherapy had an effect on survival, we used a time-dependent Cox-regression model. The use of chemotherapy in all recurrences was recorded, and their cumulative number taken as time-dependent covariates. This resulted in a HR of 1.73 (CI 1.29-2.32) for the use of chemotherapy. To examine whether this result may have been biased by a more prominent use of chemotherapy in cases with increased therapeutic pressure due to more progressive disease and therefore worse outcome, we limited the model to only include local relapses. In contrast to the initial result, a HR for chemotherapy of 0.99 (CI 0.74-1.33) was found for non-metastatic relapses.

To further examine whether the application of chemotherapy might prolong PFS after resection, we compared the median PFS with or without chemotherapy after either GTR/NTR or STR/no resection at first recurrence. In patients with GTR/NTR $(n=94)$, those treated with chemotherapy showed a longer median PFS than those who did not receive it [19.8 months (CI 15.1-28.8) vs. 15.1 months (CI 8.8-20.4)]. This also held true when correcting for the use of radiotherapy within the same relapse when comparing chemotherapy vs. no chemotherapy [21.1 months (CI 15.4-33.1) vs. 14 months (CI 8.8-39.8) in patients treated with radiotherapy and 19.3 months (CI 13.8-36.5) vs. 15.1 months (CI 6.2-NA) in patients without further radiotherapy]. In contrast, in the group of patients in whom only STR could be achieved or who underwent no surgery $(n=43)$, chemotherapy did not improve the median PFS [10.4 months (CI 7.2-16.5)] compared to a small group of seven patients who did not receive chemotherapy and had a median PFS of 20.5 months (CI 9-NA). In this group without complete resection, radiotherapy led to a notable increase in median PFS [17.5 months (CI 12.9-25.5) vs. 4.9 months (CI 3.8-16.5)], while chemotherapy did not improve survival within these subsets of patients.

Time from initial diagnosis to first recurrence severely affected survival after recurrence, as well as RR to chemotherapy applied. Patients in whom the first recurrences occurred over 24 months after first diagnosis showed improved PFS [20.5 (CI 15.3-26.5) vs. 13.3 (CI 9.9-17) months], OS [53.4 (CI 36.9-NA) vs. 24.7 (CI 20.4-43) months] and a higher rate of RR as a mean of all systemic therapies applied (33.5\% vs. 18.9\%). Additional information on survival is provided within the supplements.

\section{Chemotherapeutic agents}

In total, 40 different chemotherapeutic drugs were used in 59 separate single-drug applications or combinations. Evaluable responses could be ascertained for 140 of 236 applied chemotherapies. Table 2 shows all drugs used in at least five treatment courses which were eligible for measurement of response. The most commonly used single-drug chemotherapy was temozolomide applied as in the E-HIT-REZ 2005 protocol with 37 applications, however a RR of only $10.8 \%$ was found, with a median DOR of 0 months (CI $0 ; 0)$ and a median TTP of 2.56 months (CI 1.58; 4.7). A patient with a PF-A subtype EPN treated with monotherapeutic Temozolomide for 21 months according to the E-HIT-REZ-2005 protocol was the only patient in our cohort to in whom CR by chemotherapy alone was achieved, lasting for five years at current follow-up (Patient 7 in Fig. 1). Across all combinations of chemotherapy, etoposide was used most often (54 times), with a RR of $38.9 \%$ [median DOR: 0 months (CI 0; 5.04)], median TTP: 3.54 months (CI 1.64; 12.45). Out of all drugs used at least five separate times, sirolimus showed the best RR (50\%), the longest median DOR [1.28 months (CI 0; 5.95)] and median TTP [11.51 months (CI 4.22; 14)] across all combinations applied in nine patients. Interestingly, sirolimus seemed to show the best response when used as a monotherapy $[\mathrm{RR}=50 \%$, median $\mathrm{DOR}=2.46$ months 
Table 1 Patient characteristics at first recurrence

\begin{tabular}{|c|c|c|}
\hline Characteristic & $\mathrm{N}=138(\%)$ & \\
\hline \multicolumn{3}{|l|}{ Sex } \\
\hline Male & & $90(65.2 \%)$ \\
\hline Female & & $48(34.8 \%)$ \\
\hline \multicolumn{3}{|l|}{ Localisation at first diagnosis } \\
\hline Supratentorial & & $31(22.5 \%)$ \\
\hline Infratentorial & & $103(74.6 \%)$ \\
\hline Spinal & & $4(2.9 \%)$ \\
\hline \multicolumn{3}{|l|}{ Histological tumor grade } \\
\hline WHO ${ }^{\circ} \mathrm{II}$ & & $13(9.4 \%)$ \\
\hline WHO ${ }^{\circ} \mathrm{III}$ & & $125(90.6 \%)$ \\
\hline Molecular subgroup & $89(64)$ & \\
\hline PF-A & & $66(74.2 \%)$ \\
\hline PF-B & & $2(2.2 \%)$ \\
\hline ZFTA & & $18(20.2 \%)$ \\
\hline YAP1 & & $2(2.2 \%)$ \\
\hline MYCN & & $1(1.1 \%)$ \\
\hline Median age at first recurrence; years (IQR) & & $7.6(4.0,13.6)$ \\
\hline Median time to first recurrence, months (IQR) & & $22.8(14.4,39.9)$ \\
\hline \multicolumn{3}{|l|}{ Metastatic stage at first recurrence } \\
\hline M0 & & $74(53.6 \%)$ \\
\hline M1 & & $4(2.9 \%)$ \\
\hline M2 & & $25(18.1 \%)$ \\
\hline M3 & & $33(23.9 \%)$ \\
\hline M4 & & $2(1.4 \%)$ \\
\hline Surgery at first recurrence & $105(76)$ & \\
\hline GTR & & $62(59.0 \%)$ \\
\hline NTR & & $32(30.5 \%)$ \\
\hline STR & & $8(7.6 \%)$ \\
\hline Biopsy & & $3(2.9 \%)$ \\
\hline Radiotherapy at first recurrence & $70(51)$ & \\
\hline CSI & & $23(32.9 \%)$ \\
\hline Focal radiotherapy & & $47(67.1 \%)$ \\
\hline Median target volume dose of focal radiotherapy; Gy (IQR) & $69(50)$ & $50.4(46.0,54.0)$ \\
\hline Median target volume dose of CSI; Gy (IQR) & $22(16)$ & $35.2(35.2,35.2)$ \\
\hline Radiotherapy at initial diagnosis & & $120(87.0 \%)$ \\
\hline Median target volume dose at initial diagnosis; Gy (IQR) & & $59.4(54.0,68.0)$ \\
\hline Chemotherapy at initial diagnosis & & $108(78.3 \%)$ \\
\hline Chemotherapy at 1 st recurrence & & $99(71.7 \%)$ \\
\hline Chemotherapy at 2 nd recurrence & & $47(34.1 \%)$ \\
\hline Chemotherapy at 3 rd recurrence & & $29(21.0 \%)$ \\
\hline Chemotherapy at 4 th recurrence & & $14(10.1 \%)$ \\
\hline Chemotherapy at 5 th recurrence & & $8(5.8 \%)$ \\
\hline
\end{tabular}

$I Q R$ interquartile range, GTR gross-total resection, NTR near-total resection, STR subtotal resection, CSI craniospinal irradiation
(CI 0; 6.29) and median TTP $=14.52$ months (CI 10.65; 17.68)]. All patients treated with sirolimus had an EPN of infratentorial origin (7 PFA, 1 ZFTA, 1 unknown molecular type), data for all patients are shown in Table 3.

\section{Chemotherapy combinations}

Our analysis showed 31 combinations of at least two drugs. However, only a small fraction of three combinations were used at least five times. The three most commonly used 
Table 2 Eight most frequently used chemotherapy drugs with evaluable responses

\begin{tabular}{|c|c|c|c|c|c|c|c|c|c|}
\hline Drug & $\mathrm{n}$ & $\mathrm{CR}$ & PR & SD & $\mathrm{PD}$ & ORR $(\%)$ & $\mathrm{RR}(\%)$ & Median duration of response & Median time to progression \\
\hline Etoposide & 54 & 0 & 3 & 18 & 33 & 5.6 & 38.9 & $0(\mathrm{CI} 0 ; 5.04)$ & 3.54 (CI 1.64; 12.45) \\
\hline Temozolomide & 46 & 1 & 3 & 9 & 33 & 8.7 & 28.3 & $0(\mathrm{CI} 0 ; 0.79)$ & 2.64 (CI 1.59; 8.09) \\
\hline Trofosfamide & 30 & 0 & 1 & 10 & 19 & 3.3 & 36.7 & $0($ CI $0 ; 6.13)$ & $4(\mathrm{CI} 1.81 ; 11.44)$ \\
\hline Carboplatin & 20 & 0 & 1 & 7 & 12 & 5 & 40 & 0 (CI $0 ; 4.69)$ & 3.93 (CI $1.71 ; 12.87$ ) \\
\hline Cyclophosphamide & 13 & 0 & 0 & 5 & 8 & 0 & 38.5 & $0(\mathrm{CI} 0 ; 2.24)$ & 3.17 (CI $1.93 ; 8.97)$ \\
\hline Vincristine & 12 & 0 & 0 & 4 & 8 & 0 & 33.3 & $0(\mathrm{CI} 0 ; 2.64)$ & 3.29 (CI 2.2; 7.46) \\
\hline Sirolimus & 8 & 0 & 0 & 4 & 4 & 0 & 50 & $1.28(\mathrm{CI} 0 ; 5.95)$ & $11.51(\mathrm{CI} 4.22 ; 14)$ \\
\hline Topotecan & 7 & 0 & 0 & 0 & 7 & 0 & 0 & $0(\mathrm{CI} 0 ; 0)$ & $1.32($ CI $0.55 ; 1.61)$ \\
\hline
\end{tabular}

$n$ Number of times used, $C R$ complete remission, $P R$ partial remission, $S D$ disease stabilization, $P D$ progressive disease, $O R R$ objective response-rate, $R R$ response-rate, $C I 95 \%$ confidence-interval

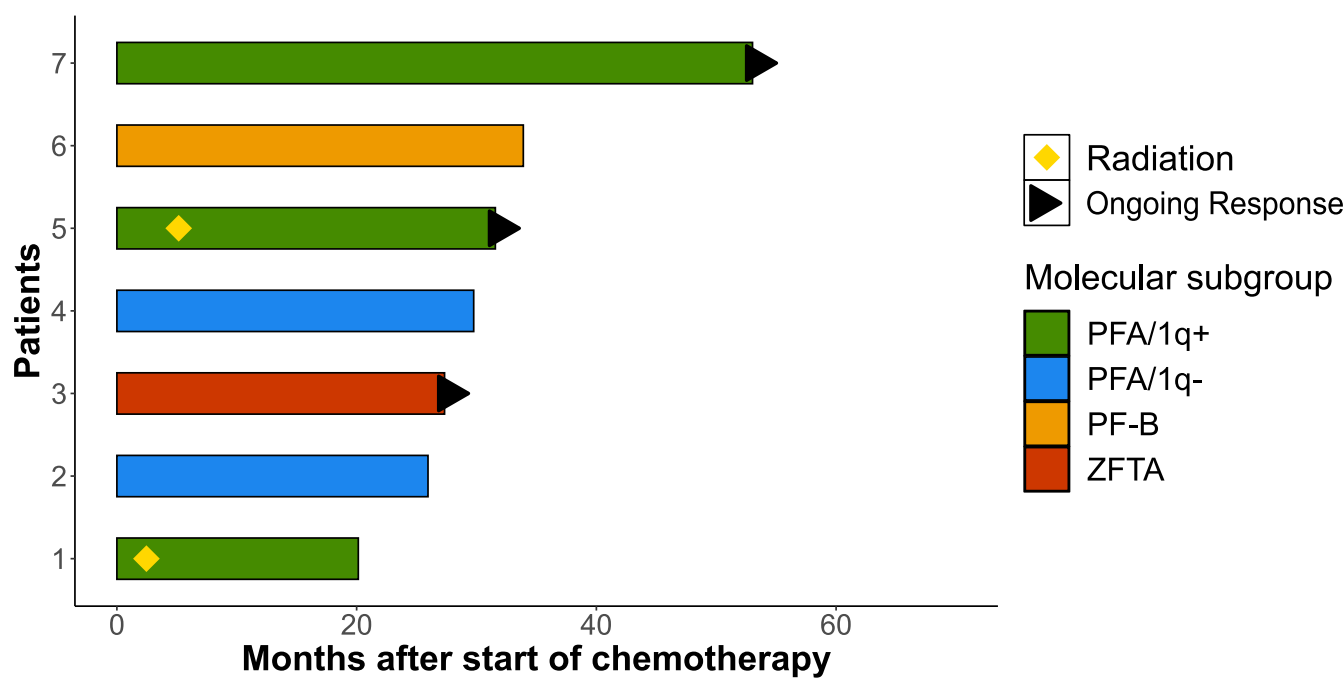

Fig. 1 Patients with subtotal or no resection at any recurrence and a PFS of over 12 months. Ongoing response denoted at date of last follow-up. Patient (P) 1: 1st recurrence, Vincristine + Cyclophosphamide + Carboplatin + Etoposide (HIT-SKK); P2: 3rd recurrence, Etoposide + Trofosfamide (HIT-REZ 2005); P3: 1st recurrence, HIT-

protocols were E-HIT-REZ 2005 (second line treatment of etoposide + trofosfamide), HIT-SKK (in chemotherapy-naive patients only; carboplatin + cyclophosphamide + etoposide + vincristine) and HIT-REZ 97 (carboplatin + etoposide). The HIT-REZ 97 protocol showed the best RR (44.4\%) and TTP $(8.93$ (CI $3.78 ; 14.22)$ months) out of these three.

\section{Chemotherapy in different molecular types}

To examine whether any chemotherapeutic drug showed an improved efficacy on OS in specific molecular EPN types, we calculated HRs regarding OS, depending on whether a specific chemotherapeutic drug was given to a patient or not during treatment of all recurrences. HR were calculated for all drugs used in at least ten patients. Table 4 lists the three drugs with the lowest HR in all patients and in the four most
SKK then Actinomycin D+Etoposide + Trofosfamide; P4: 5th recurrence, 5-FU; P5: 1st recurrence, Etoposide + Trofosfamide (HIT-REZ 2005); P6: 1st recurrence, Temozolomide, P7: 2nd recurrence, Temozolomide (HIT-REZ 2005)

common types. No significant advantage for a specific drug was found in any molecular type. However, trofosfamide and sirolimus showed a trend towards being more efficacious in PF-A tumors with or without chromosome 1q-gain, respectively. Overall, temozolomide and sirolimus showed the most improved HRs observed across all types of EPN.

\section{Chemotherapy salvage}

To investigate whether chemotherapy was able to extend survival after STR or no resection, which normally lead to severely shortened PFS and OS, we considered all relapses with incomplete resections and looked for patients treated with chemotherapy who showed a PFS of at least 12 months after start of chemotherapy. Seven patients fitting these criteria were found, with their respective swimmer plots being 


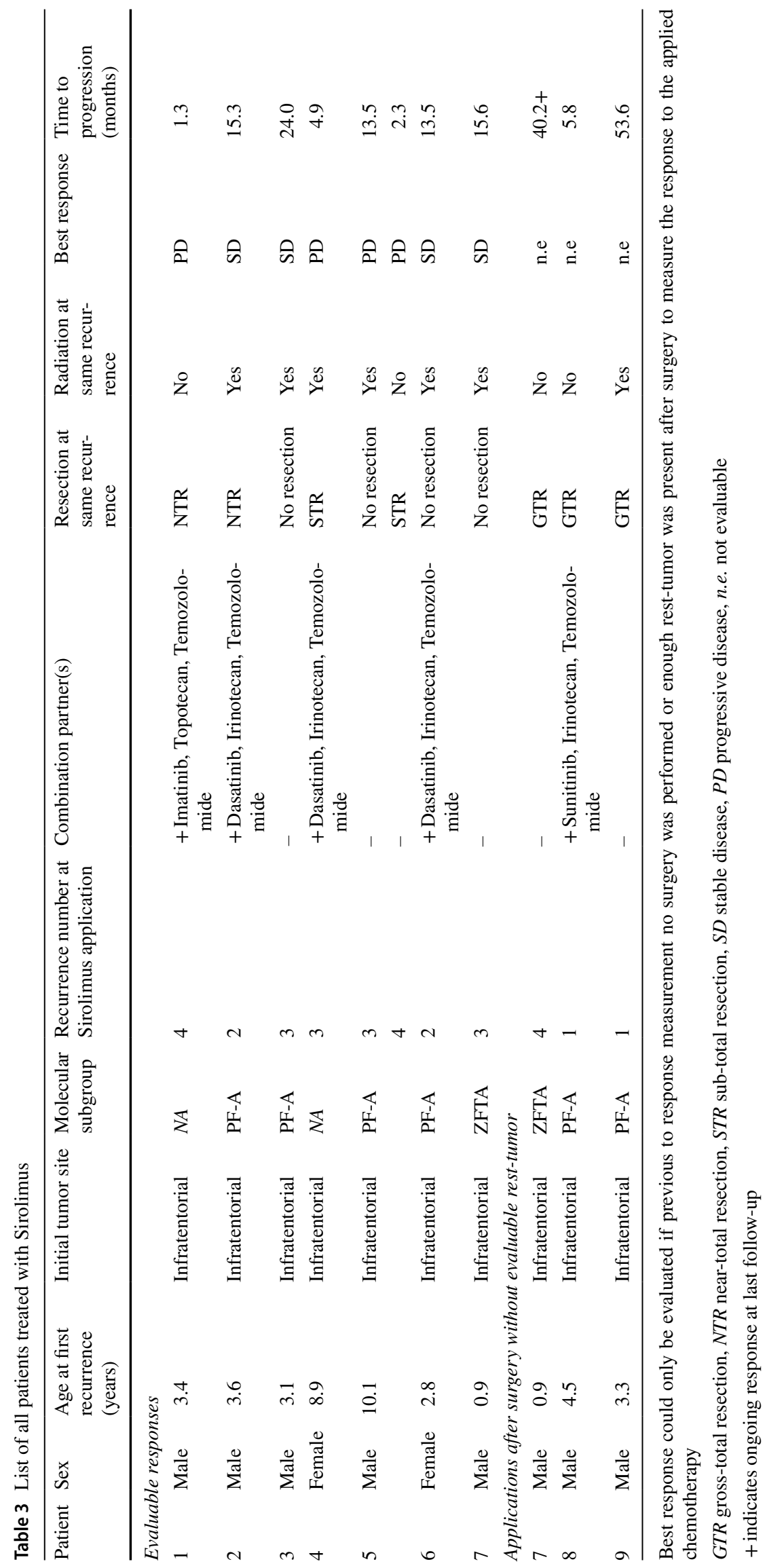


Table 4 Cox-regression comparing OS depending on whether or not a patient received specific chemotherapy drugs

\begin{tabular}{lllll}
\hline Subgroup & Chemotherapy & HR & $95 \%$-CI & p value \\
\hline All & Temozolomide & 0.7 & $(0.5-1.1)$ & 0.1 \\
& Sirolimus & 0.7 & $(0.3-1.7)$ & 0.44 \\
& Vincristine & 0.97 & $(0.5-1.7)$ & 0.93 \\
PFA & Temozolomide & 0.71 & $(0.4-1.4)$ & 0.31 \\
& Sirolimus & 0.85 & $(0.3-2.4)$ & 0.75 \\
& Trofosfamide & 0.89 & $(0.5-1.7)$ & 0.72 \\
PFA/1q+ & Trofosfamide & 0.6 & $(0.2-1.7)$ & 0.34 \\
& Cyclophosphamide & 0.7 & $(0.2-2.4)$ & 0.57 \\
& Temozolomide & 0.85 & $(0.3-2.2)$ & 0.74 \\
PFA/1q- & Sirolimus & 0.39 & $(0.1-3)$ & 0.36 \\
& Temozolomide & 0.61 & $(0.2-1.6)$ & 0.31 \\
& Trofosfamide & 1.2 & $(0.5-3.1)$ & 0.7 \\
ZFTA & Trofosfamide & 0.67 & $(0.2-2.6)$ & 0.56 \\
& Temozolomide & 0.76 & $(0.2-2.6)$ & 0.66 \\
& Cyclophosphamide & 1 & $(0.2-4.7)$ & 1 \\
\hline
\end{tabular}

$H R$ hazard ratio, $95 \%$-CI 95\% confidence-interval

shown in Fig. 1. Within these patients, three responses were still ongoing at the time of last follow-up (Patients 3, 5, and 7).

\section{Discussion}

We present a heterogenous cohort of 138 patients with recurrent EPN from two multi-institutional trials and a Germanywide registry. We analyzed the times and rates of response for different combinations and single drugs and compared the effect of chemotherapy on survival. Previous data on the systemic treatment of recurrent EPN is sparse, comprising of a mixture of clinical trials with limited case numbers and larger cohorts with diverse systemic treatments. We aim to add data from our cohort treated over the span of 20 years in Germany.

In our survival analysis we found conflicting results for the application of chemotherapy. In time-dependent Coxregression analysis, chemotherapy showed no improvement in survival regarding PFS or OS. However, in patients with GTR/NTR, PFS could be improved by chemotherapy. We also found seven patients in whom chemotherapy was able to achieve long-term survival after subtotal or no resection. As such, a subset of patients may benefit from systemic therapy, especially if other treatment options are futile. Our findings on the lack of a general survival advantage for chemotherapy is in line with previous results $[1,2,5]$.

In a small subset of seven patients with recurrences in which GTR or NTR could not be achieved - which typically show a dismal prognosis [18-20]—chemotherapy was able to induce a long-term PFS. The likelihood of this was seemingly not influenced by the molecular type, with PF-A, PF-B and ZFTA EPN falling within this subset.

Concerning responses and times to progression of specific chemotherapeutic drugs, we found sirolimus to be the most efficacious. After resections with evaluable residual tumor or no resection, we found a 50\% RR and a median TTP of 11.5 months for treatment with sirolimus. However, no cases of tumor regression under treatment with sirolimus were found in our cohort, as only disease stabilization was reached. Interestingly, patients in whom sirolimus was used as a monotherapy showed an improved RR and median TTP compared to patients in whom it was used as part of a chemotherapeutic regimen of multiple drugs, mostly the RIST-protocol (NCT01467986) [21]. Pre-clinical studies on mouse ependymoma cell-lines reported that inhibition of the mTOR-pathway can induce autophagy in EPN and showed an increased survival in mice transplanted with such cell-lines when treated with sirolimus [22]. Some data also suggest an upregulation of the mTOR-pathway within EPN of the posterior fossa, with a subset of cases showing immunohistochemical staining for phosphorylated S6 [23, 24]. Clinical data on the use of sirolimus in EPN is scarce and consists of one case-report and three phase I trials not specific to EPN, reporting on a total of five recurrent EPNs, showing success in some cases [24-27]. Our findings on sirolimus are limited by the small number of applications in our cohort $(\mathrm{n}=11)$. Additionally, it was applied more commonly in later recurrences, when generally MRI controls might be less frequent, which would result in a bias towards overstating the TTP. However, the markedly improved RR and TTP we found in our cohort may warrant future trials to evaluate its efficacy in recurrent EPN.

With the introduction of molecularly defined EPN types, there might be the possibility of a more individualized approach to its treatment. So far, clinical results on this matter are lacking $[3,12,28]$. To test whether any chemotherapeutic drugs were associated with better outcomes in specific molecular subgroups, we analyzed their influence on the OS in patients with PF-A or ZFTA EPN. We found no clearly significant advantages for the use of specific drugs in any subgroup. While specific drugs showed improved HRs in our analysis across different molecular types, no clear results could be found. Overall, larger case-numbers are needed to draw significant conclusions on potential benefits of specific chemotherapy-regimens in different EPN types.

Our results are limited by the non-randomized nature of the involved studies. While the HIT-REZ 97 and -2005 trials were conducted using pre-determined chemotherapy regimens at recurrence, further chemotherapies used after progression or relapse were chosen by local physicians in consultation with the trial office. The HIT-REZ registry includes only such chemotherapies chosen by local centers 
after consultation. This decision-making process may have led to biases on whether and which chemotherapies were chosen. For example, more intensive regimens may have been chosen in patients in whom successful long-term treatment was more likely, and therefore may show better outcomes than less intense chemotherapy chosen in palliative treatment plans. Furthermore, the measured TTP may be biased by curative vs. palliative treatment intentions, as in palliative cases and after multiple recurrences MRI and lumbar punctures may have been used more infrequently. This would lead to an overestimation of the TTP of chemotherapies especially used in patients with poor prognosis. To counteract this bias we added the DOR, which was set at 0 months for treatments if no responses than PD were found, regardless of the timing of further diagnostic tests. However, the overall low rates of response in EPN meant that the median DOR of most drugs or combinations was 0 months, making the interpretation of this parameter apart from the TTP hard.

Chemotherapy in recurrent EPN remains poorly understood. We contribute our experiences from our cohort of 138 recurrent EPN treated within Germany over the last twenty years. While we found no survival advantage for the general use of chemotherapy, we showed that in some patients, long-term tumor control via systemic treatment in absence of local therapy-options is possible. Furthermore, our data suggests that some drugs trend towards being more efficient in specific molecular types of EPN. Unfortunately, case numbers are not yet large enough to draw significant conclusions. Future pre-clinical models are offering the chance for a more individualized approach to chemotherapy and may soon influence treatment choices. While overall recurrent EPN seems to be largely resistant to chemotherapy, its use as a salvage treatment can offer improved outcome for individual patients.

Supplementary Information The online version contains supplementary material available at https://doi.org/10.1007/s11060-021-03867-8.

Acknowledgements We would like to extend our sincere gratitude to all patients and their families who participated in our study for their contribution and trust. We also thank all of our colleagues and data managers of all hospitals participating in our study for their contributions.

Author contributions All authors contributed to the study conception and design. Material preparation, data collection and analysis were performed by JEA, GF, CG, RM, MM, SR and ST. BB and MW-M provided reference radiology. BT, SD and R-DK acted as reference radiotherapists. Reference pathology was provided by TP. US, KWP, TM, SMP, OW and TP provided molecular data and performed analysis concerning targeted therapy. The first draft of the manuscript was written by JEA and all authors commented on previous versions of the manuscript. All authors read and approved the final manuscript.
Funding Open Access funding enabled and organized by Projekt DEAL. The HIT-REZ studies were supported by research grants from the German Children Cancer Foundation (Grant Nos. DKS 2006.01, DKS 2008.07, DKS 2012.03, DKS 2015.02, DKS 2017.12, DKS 2020.02) and grants to the Reference Centers for Neuroradiology and Neuropathology of the German GPOH HIT-Network from the German Children Cancer Foundation.

Data availability Anonymized data available upon reasonable request.

Code availability $\mathrm{R}$ code available upon reasonable request.

\section{Declarations}

Conflict of interest Authors declare that they have no conflict of interest.

Ethical approval All procedures in these studies involving human participants were done in accordance with the ethical standards of the institutional and national committees. All trials were conducted in accordance with the 1964 Helsinki Declaration and its later amendments or with comparable ethical standards. The institutional review boards or ethics committees of all participating centers reviewed and approved all protocols. All parents/guardians and patients, when appropriate, gave their written informed consent for data collection and analysis.

Consent to participate Written informed consent was obtained from all individual participants (patients and/or their guardians) in the study.

Consent for publication All participants (patients and/or their guardians) of the included studies gave their informed consent to publish the data obtained in an anonymized publication.

Open Access This article is licensed under a Creative Commons Attribution 4.0 International License, which permits use, sharing, adaptation, distribution and reproduction in any medium or format, as long as you give appropriate credit to the original author(s) and the source, provide a link to the Creative Commons licence, and indicate if changes were made. The images or other third party material in this article are included in the article's Creative Commons licence, unless indicated otherwise in a credit line to the material. If material is not included in the article's Creative Commons licence and your intended use is not permitted by statutory regulation or exceeds the permitted use, you will need to obtain permission directly from the copyright holder. To view a copy of this licence, visit http://creativecommons.org/licenses/by/4.0/.

\section{References}

1. Byer L, Kline CN, Coleman C, Allen IE, Whitaker E, Mueller S (2019) A systematic review and meta-analysis of outcomes in pediatric, recurrent ependymoma. J Neurooncol 144:445-452. https://doi.org/10.1007/s11060-019-03255-3

2. Ritzmann TA, Rogers HA, Paine SML, Storer LCD, Jacques TS, Chapman RJ, Ellison D, Donson AM, Foreman NK, Grundy RG (2020) A retrospective analysis of recurrent pediatric ependymoma reveals extremely poor survival and ineffectiveness of current treatments across central nervous system locations and molecular subgroups. Pediatr Blood Cancer 67:e28426. https:// doi.org/10.1002/pbc.28426 
3. Massimino M, Barretta F, Modena P, Witt H, Minasi S, Pfister SM, Pajtler KW, Antonelli M, Gandola L, Luisa Garre M, Bertin D, Mastronuzzi A, Mascarin M, Quaglietta L, Viscardi E, Sardi I, Ruggiero A, Pollo B, Buccoliero A, Boschetti L, Schiavello E, Chiapparini L, Erbetta A, Morra I, Gessi M, Donofrio V, Patriarca C, Giangaspero F, Johann P, Buttarelli FR (2021) Second series by the Italian association of pediatric hematology and oncology of children and adolescents with intracranial ependymoma: an integrated molecular and clinical characterization with a longterm follow-up. Neuro Oncol 23:848-857. https://doi.org/10.1093/ neuonc/noaa257

4. Junger ST, Mynarek M, Wohlers I, Dorner E, Muhlen AZ, VelezChar N, von Hoff K, Rutkowski S, Warmuth-Metz M, Kortmann RD, Timmermann B, Rahmann S, Klein-Hitpass L, von Bueren AO, Pietsch T (2019) Improved risk-stratification for posterior fossa ependymoma of childhood considering clinical, histological and genetic features - a retrospective analysis of the HIT ependymoma trial cohort. Acta Neuropathol Commun 7:181. https:// doi.org/10.1186/s40478-019-0820-5

5. Zacharoulis S, Ashley S, Moreno L, Gentet JC, Massimino M, Frappaz D (2010) Treatment and outcome of children with relapsed ependymoma: a multi-institutional retrospective analysis. Childs Nerv Syst 26:905-911. https://doi.org/10.1007/ s00381-009-1067-4

6. Bouffet E, Hawkins CE, Ballourah W, Taylor MD, Bartels UK, Schoenhoff N, Tsangaris E, Huang A, Kulkarni A, Mabbot DJ, Laperriere N, Tabori U (2012) Survival benefit for pediatric patients with recurrent ependymoma treated with reirradiation. Int J Radiat Oncol Biol Phys 83:1541-1548. https://doi.org/10. 1016/j.ijrobp.2011.10.039

7. Tsai JW, Manoharan N, Alexandrescu S, Zimmerman MA, Scully J, Chordas C, Clymer J, Wright KD, Filbin M, Ullrich NJ, Marcus KJ, Haas-Kogan D, Chi SN, Bandopadhayay P, Yeo KK (2021) Outcomes after first relapse of childhood intracranial ependymoma. Pediatr Blood Cancer 68:e28930. https://doi.org/ $10.1002 /$ pbc. 28930

8. Adolph JE, Fleischhack G, Mikasch R, Zeller J, Warmuth-Metz M, Bison B, Mynarek M, Rutkowski S, Schuller U, von Hoff K, Obrecht D, Pietsch T, Pfister SM, Pajtler KW, Witt O, Witt H, Kortmann RD, Timmermann B, Krauss J, Fruhwald MC, Faldum A, Kwiecien R, Bode U, Tippelt S (2021) Local and systemic therapy of recurrent ependymoma in children and adolescents: short- and long-term results of the E-HIT-REZ 2005 study. Neuro Oncol 23:1012-1023. https://doi.org/10.1093/neuonc/noaa276

9. Siffert J, Allen JC (1998) Chemotherapy in recurrent ependymoma. Pediatr Neurosurg 28:314-319. https://doi.org/10.1159/ 000028669

10. Bouffet E, Capra M, Bartels U (2009) Salvage chemotherapy for metastatic and recurrent ependymoma of childhood. Childs Nerv Syst 25:1293-1301. https://doi.org/10.1007/s00381-009-0883-x

11. Pajtler KW, Witt H, Sill M, Jones DT, Hovestadt V, Kratochwil F, Wani K, Tatevossian R, Punchihewa C, Johann P, Reimand J, Warnatz HJ, Ryzhova M, Mack S, Ramaswamy V, Capper D, Schweizer L, Sieber L, Wittmann A, Huang Z, van Sluis P, Volckmann R, Koster J, Versteeg R, Fults D, Toledano H, Avigad S, Hoffman LM, Donson AM, Foreman N, Hewer E, Zitterbart K, Gilbert M, Armstrong TS, Gupta N, Allen JC, Karajannis MA, Zagzag D, Hasselblatt M, Kulozik AE, Witt O, Collins VP, von Hoff K, Rutkowski S, Pietsch T, Bader G, Yaspo ML, von Deimling A, Lichter P, Taylor MD, Gilbertson R, Ellison DW, Aldape K, Korshunov A, Kool M, Pfister SM (2015) Molecular classification of ependymal tumors across all CNS compartments, histopathological grades, and age groups. Cancer Cell 27:728-743. https://doi.org/10.1016/j.ccell.2015.04.002

12. Mack SC, Pajtler KW, Chavez L, Okonechnikov K, Bertrand KC, Wang X, Erkek S, Federation A, Song A, Lee C, Wang
X, McDonald L, Morrow JJ, Saiakhova A, Sin-Chan P, Wu Q, Michaelraj KA, Miller TE, Hubert CG, Ryzhova M, Garzia L, Donovan L, Dombrowski S, Factor DC, Luu B, Valentim CLL, Gimple RC, Morton A, Kim L, Prager BC, Lee JJY, Wu X, Zuccaro J, Thompson Y, Holgado BL, Reimand J, Ke SQ, Tropper A, Lai S, Vijayarajah S, Doan S, Mahadev V, Minan AF, Grobner SN, Lienhard M, Zapatka M, Huang Z, Aldape KD, Carcaboso AM, Houghton PJ, Keir ST, Milde T, Witt H, Li Y, Li CJ, Bian XW, Jones DTW, Scott I, Singh SK, Huang A, Dirks PB, Bouffet E, Bradner JE, Ramaswamy V, Jabado N, Rutka JT, Northcott PA, Lupien M, Lichter P, Korshunov A, Scacheri PC, Pfister SM, Kool M, Taylor MD, Rich JN (2018) Therapeutic targeting of ependymoma as informed by oncogenic enhancer profiling. Nature 553:101-105. https://doi.org/10.1038/nature25169

13. Arabzade A, Zhao Y, Varadharajan S, Chen HC, Jessa S, Rivas B, Stuckert AJ, Solis M, Kardian A, Tlais D, Golbourn BJ, Stanton AJ, Chan YS, Olson C, Karlin KL, Kong K, Kupp R, Hu B, Injac SG, Ngo M, Wang PR, De Leon LA, Sahm F, Kawauchi D, Pfister SM, Lin CY, Hodges HC, Singh I, Westbrook TF, Chintagumpala MM, Blaney SM, Parsons DW, Pajtler KW, Agnihotri S, Gilbertson RJ, Yi J, Jabado N, Kleinman CL, Bertrand KC, Deneen B, Mack SC (2021) ZFTA-RELA dictates oncogenic transcriptional programs to drive aggressive supratentorial ependymoma. Cancer Discov. https://doi.org/10.1158/2159-8290.CD-20-1066

14. Ferguson SD, Zhou S, Xiu J, Hashimoto Y, Sanai N, Kim L, Kesari S, de Groot J, Spetzler D, Heimberger AB (2018) Ependymomas overexpress chemoresistance and DNA repair-related proteins. Oncotarget 9:7822-7831. https://doi.org/10.18632/oncot arget. 23288

15. Sabnis DH, Storer LCD, Liu JF, Jackson HK, Kilday JP, Grundy RG, Kerr ID, Coyle B (2019) A role for ABCB1 in prognosis, invasion and drug resistance in ependymoma. Sci Rep 9:10290. https://doi.org/10.1038/s41598-019-46700-z

16. R Core Team (2020) R: a language and environment for statistical computing. R Foundation for Statistical Computing, Vienna, Austria. https://www.R-project.org/

17. RStudio Team (2020) RStudio: Integrated Development for R. RStudio, PBC, Boston, MA. http://www.rstudio.com/

18. Pollack IF, Gerszten PC, Martinez AJ, Lo KH, Shultz B, Albright AL, Janosky J, Deutsch M (1995) Intracranial ependymomas of childhood: long-term outcome and prognostic factors. Neurosurgery 37:655-666. https://doi.org/10.1227/00006123-19951000000008 (discussion 666-657)

19. Messahel B, Ashley S, Saran F, Ellison D, Ironside J, Phipps K, Cox T, Chong WK, Robinson K, Picton S, Pinkerton CR, Mallucci C, Macarthur D, Jaspan T, Michalski A, Grundy RG, Children's Cancer Leukaemia Group Brain Tumour Cells (2009) Relapsed intracranial ependymoma in children in the UK: patterns of relapse, survival and therapeutic outcome. Eur J Cancer 45:1815-1823. https://doi.org/10.1016/j.ejca.2009.03.018

20. Gupta T, Maitre M, Gupta P, Krishnatry R, Chatterjee A, Moiyadi A, Shetty P, Singh V, Chinnaswamy G, Epari S, Sahay A, Patil V, GodaSastri J (2020) Extent of re-excision, sequence/timing of salvage re-irradiation, and disease-free interval impact upon clinical outcomes in recurrent/progressive ependymoma. J Neurooncol 147:405-415. https://doi.org/10.1007/s11060-020-03434-7

21. Nonnenmacher L, Westhoff MA, Fulda S, Karpel-Massler G, Halatsch ME, Engelke J, Simmet T, Corbacioglu S, Debatin KM (2015) RIST: a potent new combination therapy for glioblastoma. Int J Cancer 136:E173-187. https://doi.org/10.1002/ijc.29138

22. Dewi FRP, Jiapaer S, Kobayashi A, Hazawa M, Ikliptikawati DK, Hartono SH, Nakada M, Wong RW (2021) Nucleoporin TPR (translocated promoter region, nuclear basket protein) upregulation alters MTOR-HSF1 trails and suppresses autophagy induction in ependymoma. Autophagy 17:1001-1012. https://doi.org/ $10.1080 / 15548627.2020 .1741318$ 
23. Wang G, Jia Y, Ye Y, Kang E, Chen H, Wang J, He X (2021) Identification of key methylation differentially expressed genes in posterior fossa ependymoma based on epigenomic and transcriptome analysis. J Transl Med 19:174. https://doi.org/10.1186/ s12967-021-02834-1

24. Bowers DC, Kucejova B, Margraf L, Gargan L, Brugarolas J (2011) mTORC1 activation in childhood ependymoma and response to sirolimus. J Neurooncol 103:797-801. https://doi. org/10.1007/s11060-010-0455-7

25. Piha-Paul SA, Shin SJ, Vats T, Guha-Thakurta N, Aaron J, Rytting M, Kleinerman E, Kurzrock R (2014) Pediatric patients with refractory central nervous system tumors: experiences of a clinical trial combining bevacizumab and temsirolimus. Anticancer Res 34:1939-1945

26. Becher OJ, Gilheeney SW, Khakoo Y, Lyden DC, Haque S, De Braganca KC, Kolesar JM, Huse JT, Modak S, Wexler LH, Kramer K, Spasojevic I, Dunkel IJ (2017) A phase I study of perifosine with temsirolimus for recurrent pediatric solid tumors. Pediatr Blood Cancer. https://doi.org/10.1002/pbc.26409

27. Qayed M, Cash T, Tighiouart M, MacDonald TJ, Goldsmith KC, Tanos R, Kean L, Watkins B, Suessmuth Y, Wetmore C, Katzenstein HM (2020) A phase I study of sirolimus in combination with metronomic therapy (CHOAnome) in children with recurrent or refractory solid and brain tumors. Pediatr Blood Cancer 67:e28134. https://doi.org/10.1002/pbc.28134

28. Wu J, Armstrong TS, Gilbert MR (2016) Biology and management of ependymomas. Neuro Oncol 18:902-913. https://doi.org/ 10.1093/neuonc/now016

Publisher's Note Springer Nature remains neutral with regard to jurisdictional claims in published maps and institutional affiliations. 